Article

\title{
Semi-Automatic System for Land Cover Change Detection Using Bi-Temporal Remote Sensing Images
}

\author{
ZhiYong Lv ${ }^{1, *}$, WenZhong Shi ${ }^{2, *}$, XiaoCheng Zhou ${ }^{3}$ and Jón Atli Benediktsson ${ }^{4}$
}

1 School of Computer Science and Engineering, Xi'an University of Technology, Xi'an 710048, China

2 Department of Land Surveying and Geo-Informatics, The Hong Kong Polytechnic University, Hung Hom, Hong Kong, China

3 Key Laboratory of Spatial Data Mining \& Information Sharing of Ministry of Education, Fuzhou University, Fuzhou 350116, China; zhouxc@fzu.edu.cn

4 Faculty of Electrical and Computer Engineering, University of Iceland, Reykjavik IS 107, Iceland; benedikt@hi.is.

* Correspondence: Lvzhiyong_fly@hotmail.com (Z.L.); lswzshi@polyu.edu.hk (W.S.); Tel.: +86-158-2967-3435 (Z.L.)

Received: 18 August 2017; Accepted: 23 October 2017; Published: 31 October 2017

\begin{abstract}
Change detection is an increasingly important research topic in remote sensing application. Previous studies achieved land cover change detection (LCCD) using bi-temporal remote sensing images. However, many widely used methods detected change depending on a series of parameters, and determining parameters is time-consuming. Furthermore, numerous methods are data-dependent. Therefore, their degree of automation should be improved significantly. Three techniques, which consist of a semi-automatic change detection system, are proposed for LCCD to overcome the abovementioned drawbacks. The three techniques are as follows: (1) change magnitude image (CMI) noise reduction is based on Gaussian filter (GF), which is coupled with OTSU for reducing CMI noise automatically using an iterative optimization strategy; (2) a method based on histogram curve fitting is suggested to predict the threshold range for parameter determination; and (3) a modified region growing algorithm is built for iteratively constructing the final change detection map. The detection accuracies of the proposed system are investigated through four experiments with different bi-temporal image scenes. Compared with several widely used change detection methods, the proposed system can be applied to detect land cover change with high accuracy and flexibility. This work is an attempt to provide a change detection system that is compatible with remote sensing images with high and median-low spatial resolution.
\end{abstract}

Keywords: semi-automatic change detection system; land cover change detection; remote sensing images

\section{Introduction}

Land cover change detection (LCCD) plays an increasingly important role in various practical applications, such as urban land resource management, urban expansion, and damage assessment [1-10]. With the development of remote sensing techniques, obtaining remote sensing images over a certain area at different times is convenient. Therefore, the land cover change on earth's surface can be detected through bi-temporal remote sensing images [1,11-13].

In recent years, various methods based on remote sensing images, such as image difference [14,15], image ratio [11], change vector analysis (CVA) [16,17], and principal component analysis-based change detection methods (CD_PCA_Kmeans) [1], have been developed. In addition, pixel-based post-classification change detection can provide the "from to" change information compared with the binary change detection technique [18-22]. Most of these methods only focus on the remote sensing 
images of median-low spatial resolution, although these methods have been applied in practice. Furthermore, several parameters should be determined for generating a binary change detection map (BCDM), and parameter determination is experience-dependent and time-consuming [23].

In addition to the abovementioned methods, remote sensing image with high spatial resolution can be obtained conveniently through satellite or aerial platforms and can capture more ground details than remote sensing images with median-low spatial resolution. However, the high spatial resolution does not mean higher classification or change detection accuracy than median-low spatial resolution images due to the wide intra-class and minimal inter-class variances in the remote sensing images of high spatial resolution [24,25]. Therefore, the spatial features of high spatial resolution image, such as the features proposed in Semi_FCM [3], Markov random field [26-28], morphological profiles [29], and multi-resolution level set (MLS) [2], are considered for change detection to solve the abovementioned problem. Contextual feature extraction typically depends on various parameters, such as size, shape, direction [30], and training samples, despite improving the detection performance and accuracy. Furthermore, the contextual feature extraction algorithm is typically complex, and designing these algorithms depends considerably on experience [31,32].

In recent years, numerous remote sensing images have been obtained conveniently, and LCCD based on remote sensing images are increasingly required [33,34]. Numerous methods, such as hierarchical unsupervised change detection method [35], urban area change detection [36], and unsupervised change detection based on morphological profiles [13,37], have been developed with the increase in data availability. However, some methods have been insufficient in terms of practical application, especially for inexperienced practitioners. Therefore, automatic or semi-automatic methods should be developed given the increasing requirements of change detection based on various images.

As mentioned previously, opportunities for improvements in terms of accuracy and usability of the LCCD system still exist despite exerting considerable efforts to develop the change detection techniques for remote sensing images. For example, few methods are generally used for change detection of median-low and high spatial resolution images. However, various detection targets in remote sensing images have different appropriate observational spatial resolutions [38,39]. Remote sensing images with various spatial resolutions are required for LCCD. Therefore, a change detection system with high generality should be developed, indicating suitability for remote sensing images with high and low-resolution remote sensing images. In addition, the degree of automation for practical application should be improved.

In this work, we proposed a change detection system with a nearly automatic degree and high generality. The proposed system consists of three proposed techniques: (1) automatic noise reduction based on the Gaussian filter (GF); (2) parameter determination assistance with threshold range prediction (TRP); and (3) a modified change detection region growing algorithm (RGA) for optimizing the initial change detection result. The proposed system is data-independent and nearly automatic. Therefore, the proposed system is capable of detecting land cover change semi-automatically and has a high generality.

We conducted the experiments on four image scenes to evaluate the effectiveness of the proposed system. Two images are real multitemporal aerial datasets with a very high resolution ( $0.5 \mathrm{~m}$ per pixel) for landslide detection. The other two images, captured by Landsat-7 and Landsat-5, are open testing datasets with a resolution of $30 \mathrm{~m}$ per pixel. Further details are presented in the Experimental Section.

This rest of this paper is organized as follows. Section 2 introduces the proposed system. Section 3 describes the datasets and experimental comparisons. Section 4 discusses the experiments. Section 5 presents the conclusion. 


\section{Method}

The two co-registered remote sensing images are $X_{1}=\left\{x_{1}(i, j), 1 \leq i \leq H, 1 \leq j \leq W\right\}$ and $X_{2}=\left\{x_{2}(i, j), 1 \leq i \leq H, 1 \leq j \leq W\right\}$, where $\mathrm{H}$ and $\mathrm{W}$ are the height and width of the image, respectively, that are acquired over the same area at different times $t_{1}$ and $t_{2}$. In Figure 1 , the proposed system is composed of three main blocks intended for the following: (1) noise reduction; (2) parameter determination; and (3) improvement of change detection result. To achieve these goals, the corresponding approaches are labeled (1), (2), and (3) in Figure 1. These approaches are described in details in subsequent sections.

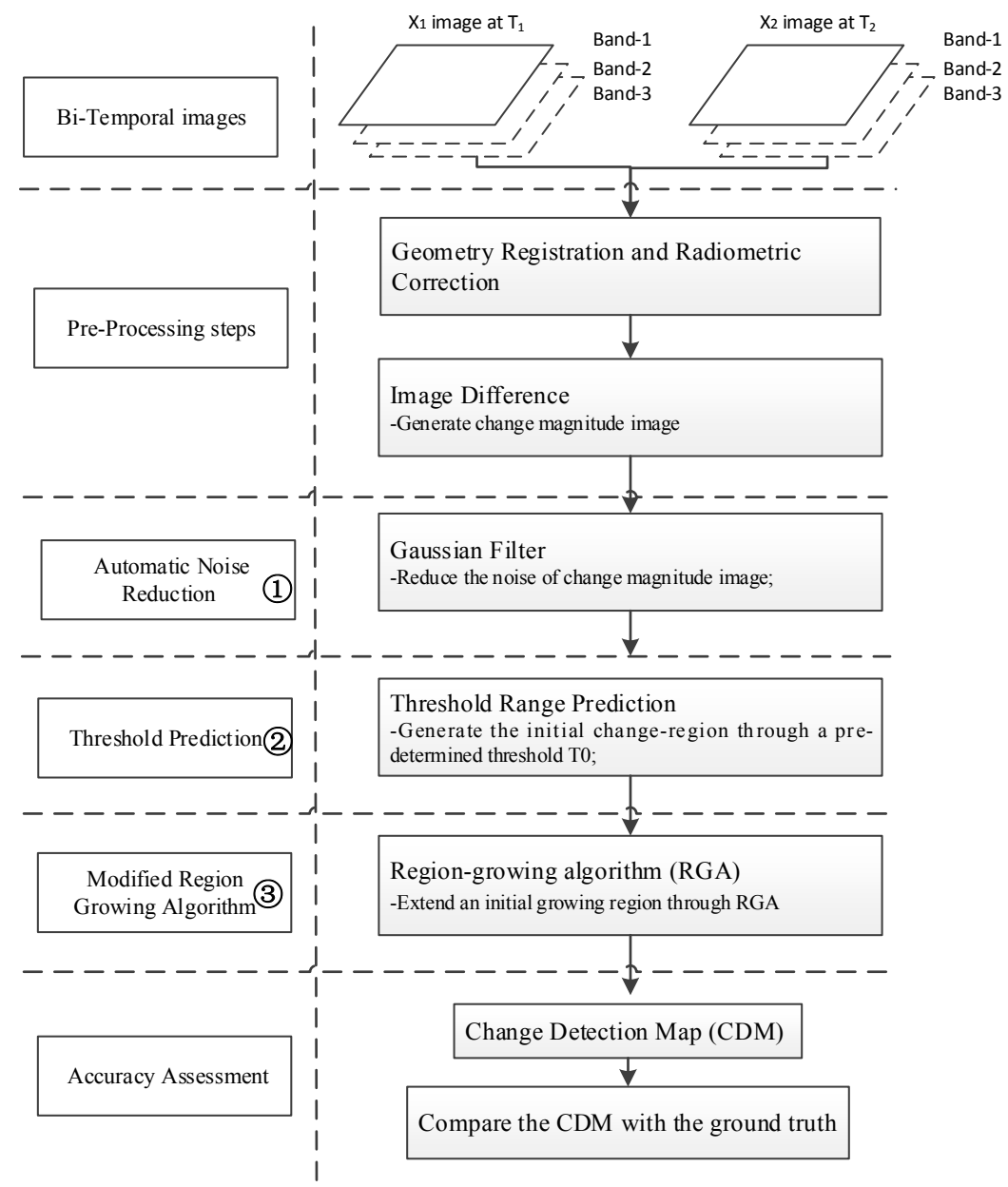

Figure 1. Processing chain of the proposed change detection system.

The preprocessing of bi-temporal images plays an important role in change detection [40-42]. In the proposed system, geometry co-registration and radiometric correction are conducted using Erdas 8.7 software. In addition, image difference, which is one of the most simple and popular methods, is adopted to generate the change magnitude image (CMI) $[3,43]$. Moreover, the change magnitude of image difference is defined as $\mathrm{d}(\mathrm{i}, \mathrm{j})=\left\|x_{i j}^{t_{1}}-x_{i j}^{t_{2}}\right\|$, where $x_{i j}^{t_{1}}$ and $x_{i j}^{t_{2}}$ are the gray pixel values obtained at times $t_{1}$ and $t_{2}$, correspondingly, and $i$ and $j$ are the row and column positions, respectively. A large value of $d(i, j)$ typically indicates that the probability of change at position $(i, j)$ is high. Thus, a bright pixel obtained in image difference normally corresponds to a high probability of change. However, multitemporal images for change detection are typically different in radiation, and the difference is caused by different atmospheric conditions, solar angles, and soil moisture. Therefore, obtaining a reliable change magnitude image is necessary. 


\subsection{Automatic Noise Reduction Based on GF}

As mentioned previously, $\mathrm{CMI}$ is usually corrupted by noise and resulted in an unreliable CMI. In this section, it considers that GF is frequently used to reduce the noise in the original image or intermediate results, as discussed in previous studies [44-46]. However, the common drawback of using GF is the requirement for "prior" knowledge about the amount of noise corruption, although Gaussian-like noise distribution is frequently encountered in acquired data [47]. This knowledge is valuable for the optimal choice of parameter. However, this information is frequently unavailable in practical applications. Therefore, an automatic noise reduction approach should be developed. In this section, an automatic noise reduction approach based on GF and OTSU [48,49] is proposed to further refine the noise in CMI. The flowchart of this proposed approach is illustrated in Figure 2, and its principle is explained as follows.

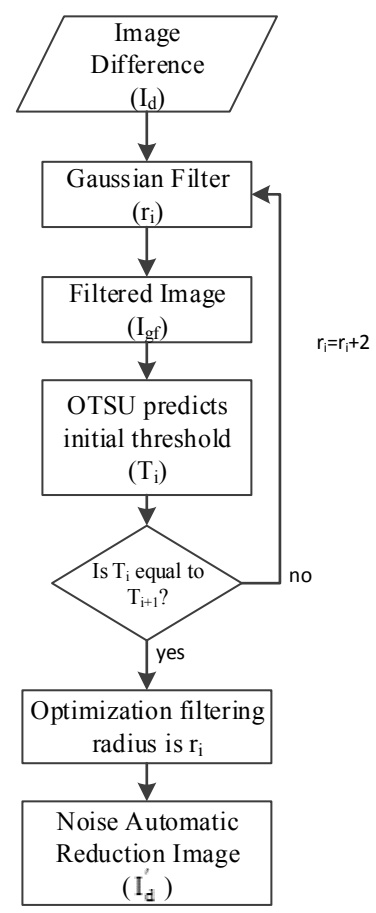

Figure 2. Automatic Noise Reduction method based on Gaussian filter (GF) and OTSU.

In the proposed automatic noise reduction method, the parameter, which is the filter radius (r) of GF, is tuned by a gradual procedure that considers the relationship between the filtered image and its corresponding OTSU-binary result. In the proposed approach, $\mathrm{r}$ is increased from small to large gradually, and each corresponding filtered image is automatically divided into a binary image through OTSU. The corresponding $\mathrm{r}$ can be considered the optimized filtered radius when the previous predicted binary threshold $\left(T_{i}\right)$ is equal to the current threshold $\left(T_{i+1}\right)$ because OTSU predicts an optimal threshold by maximizing the two-class variance [49]. The two classes are changed (foreground) and unchanged (background) pixels, especially for change detection. If the filtered result with an increasing $r$ will not vary with the binary threshold obtained by OTSU when combining GF and OTSU comprehensively, then the current ( $\mathrm{r}$ ) can smoothen the noise optimally and provide a stable performance of changed and unchanged pixels for an input CMI.

Based on the above principles, the details of the proposed technique are presented in Figure 2, as follows: (1) the GF is adopted to smoothen the CMI with an initial filtering radius $r_{i}$; the filtered image is $I_{g f}$; (2) OTSU is adopted to predict a binary threshold $\left(T_{i}\right)$ based on the $I_{g f} ;(3)$ an iteration is presented, $r_{i}$ is updated by the value of $r_{i}+2$, and the updated filtering radius is used to filter the image difference $\left(I_{d}\right) ;(4)$ in the next iteration, new threshold $\left(T_{i}+1\right)$ is obtained by OTSU based on GF 
with updated filtered radius; and (5) this iteration is terminated when the two predicted thresholds are equal, the optimal filtered radius of GF is determined, and the optimal filtered image $\left(\mathrm{I}_{\mathrm{d}}^{\prime}\right)$ is generated.

\subsection{Threshold Range Prediction (TRP) for Generating the Initial Change Detection Region}

Obtaining the change region through binary threshold based on filtered change magnitude image (FCMI) remains difficult, although the first proposed technique can reduce much noise in CMI. Furthermore, several previous studies demonstrate that determining a threshold for dividing the CMI into a BCDM is time-consuming and experience-dependent [23,50-53]. Therefore, the threshold range should be predicted automatically, especially for inexperienced practitioners. The flowchart of the second proposed technique is illustrated in Figure 3.

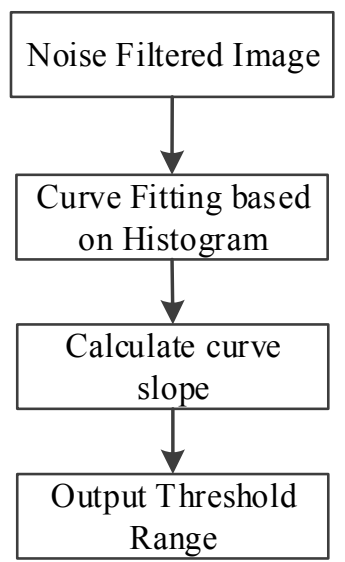

Figure 3. Flowchart of threshold range prediction (TRP).

In Figure 3, several key points are worth noting: First, curve fitting based on the histogram is conducted on the filtered image; in this study, moving least squares (MLS) is adopted as the curve fitting method [54]. MLS is used as a ten-order polynomial function to fit points and describe the trends of pixel distribution in the proposed TRP. Second, if $f(x)$ is assumed as the curve fitting function, then $S(x)$ is defined as the slope value function; the value of $S(x)$ can be calculated by the first derivative of $f(x)$. Finally, a window sliding technique is used to detect the top and bottom points at the curve of $\mathrm{S}(\mathrm{x})$; further details on this technique can be tracked in [55].

Specifically, the schematic of the proposed TRP presented in Figure 4 indicates that the pixels in FCMI are observed as "changed pixels-uncertain pixels-unchanged pixels". The noise error induced by misregistration distributes along the edge between the changed and unchanged areas [56-59]; the "uncertain pixels" distribute between the changed and unchanged areas. However, the difference between the three kinds of pixels is fuzzy and uncertain in a practical application. The distribution trend of pixels should be depicted to obtain the threshold range. Therefore, firstly, the distribution of pixels in FCMI is depicted using a histogram technique, such as in [14]. Then, a simple and classical curve fitting technique, namely, MLS method, is adopted to smoothen the noise and depict the distributed trends accurately [54]. Finally, the slope value of the curve fitting line is calculated by using a derivative, and the bottom and top values related to the threshold range can be captured.

Two examples using the FCMI of Mexico and Sardinia Island (Italy) datasets to illustrate the performance of the proposed TRP are depicted in Figure 5. The yellow pixels in Figure 5 are within the predicted range obtained by the proposed technique. The yellow pixels are distributed around the bright pixels, which indicate a high possibility of change. 


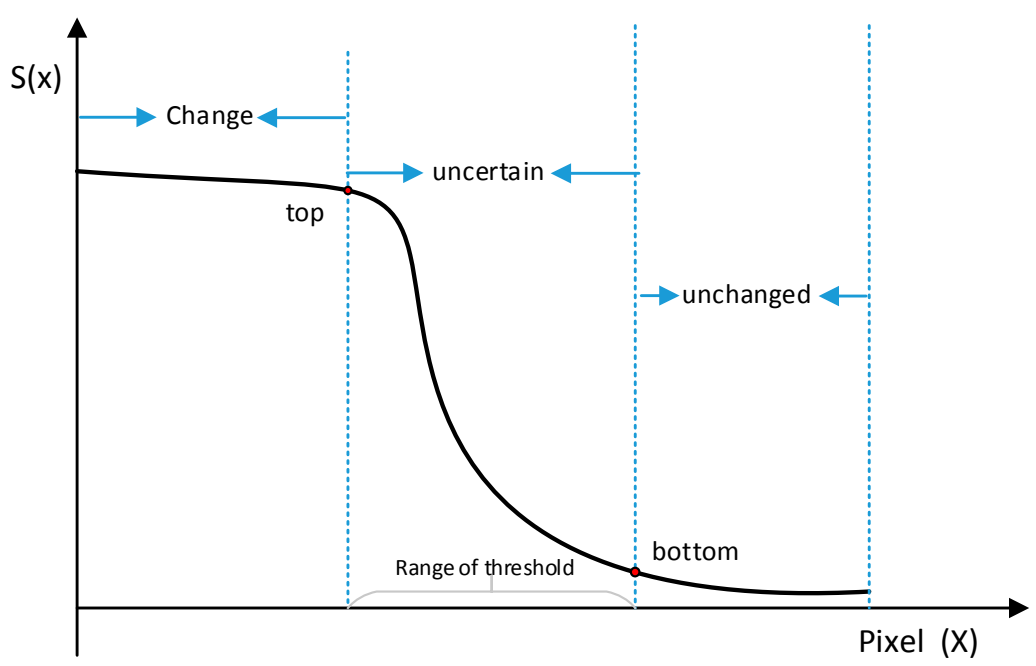

Figure 4. Schematic of the TRP technique for predicting the threshold range $(S(x)$ is the slope value, and $x$ is the pixel value of the filtered change magnitude image (FCMI).

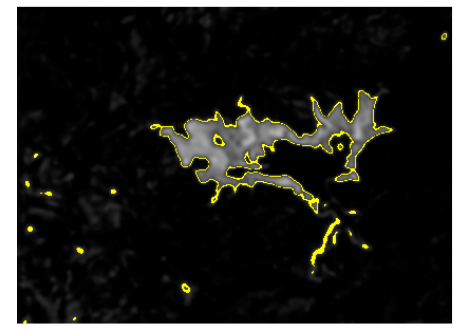

(a)

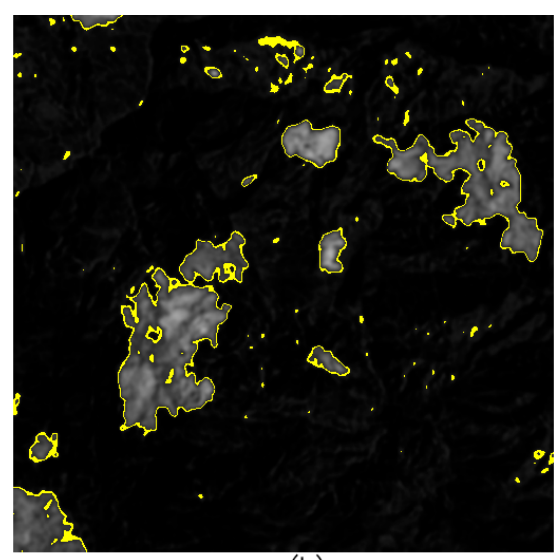

(b)

Figure 5. Distribution of pixels that are within the threshold range obtained by the TRP (the yellow pixels are the uncertain pixels; the value of these uncertain pixels are within the proposed TRP; the bright pixels are the pixels with high change probability, and the black pixels are the unchanged pixels):

(a) FCMI of Sardinia Island datasets; and (b) FCMI of Mexico datasets.

As discussed in previous studies, threshold determination is performed by using empirical strategies or manual trial-and-error procedures, which are time-consuming and affect the accuracy and reliability of change detection [60-62]. Therefore, the threshold range should be predicted for determining a rational threshold. Compared with several existing threshold decision processes for change detection $[14,26,27,63]$, the novelty of the proposed TRP is that it can automatically determine the threshold range for selecting a binary threshold. Moreover, the process of the proposed TRP is driven by the data itself and is independent of any assumption or prior knowledge.

\subsection{Modified RGA for Land Cover Change Detection (LCCD)}

Opportunities for improving the detection accuracy and performance still exist, although threshold range assistance is valuable for selecting a rational threshold for dividing the FCMI into a BCDM in terms of convenience. Therefore, a modified RGA is proposed in this section as the third technique in the proposed framework to improve the performance of change detection. The flowchart of the RGA is presented in Figure 6. 


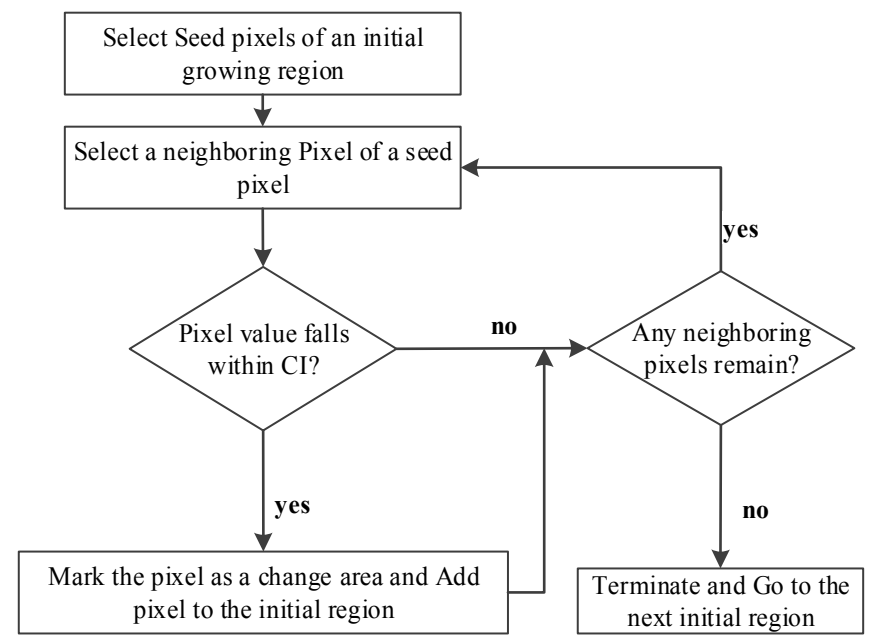

Figure 6. Proposed modified region growing algorithm (RGA).

In BCDM, the spatial change pixels continuously construct a changing area, namely, "initial growing region (IGR)", in the proposed system. First, a single changed or unchanged pixel in the $\mathrm{BCDM}$ is observed as a noise pixel that can be removed automatically. To improve the performance of the BCDM further, the FCMI and BCDM are overlaid together, and the pixels for the FCMI within an IGR are used to build a corresponding credible interval (CI): $\left[m_{r}-\delta_{r}, m_{r}+\delta_{r}\right]$, where $m_{r}$ and $\delta_{r}$ are the mean value and standard deviation of region $r$, respectively. The pixels on the boundary of $r$ are seed pixels, as the green pixels displayed in Figure $7 \mathrm{~b}$. Similar gray presents the adjacent change probability or ground material in Figure 7. If the value of a seed pixel is within the $\mathrm{CI}$, then the pixel will be marked as "changed", and this iteration will be terminated until no pixel can be found and can meet this condition. An example of the proposed RGA is demonstrated in Figure 7 to clarify this growing procedure.

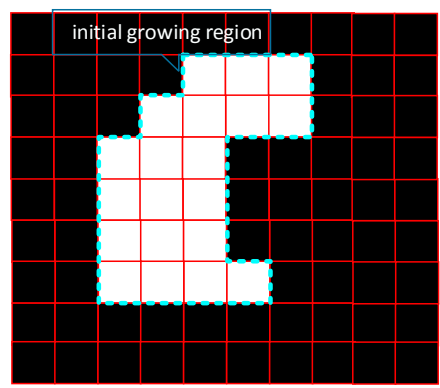

(a)

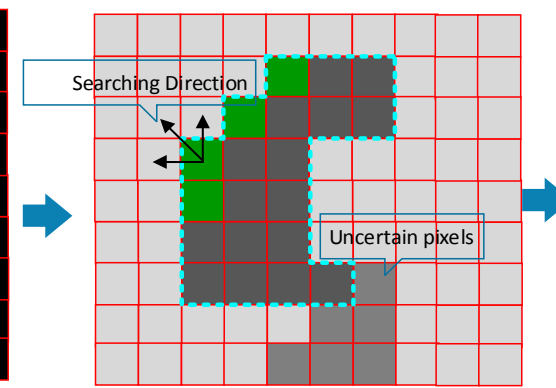

(b)

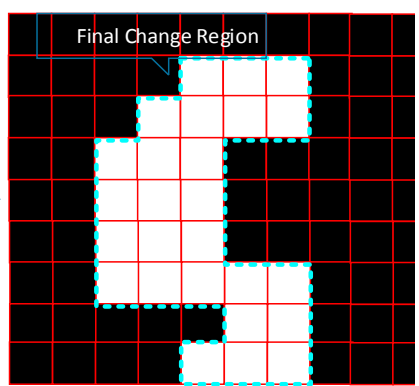

(c)

Figure 7. Example of the modified RGA: (a) IGR represented by dotted lines in the BCDM; (b) seed pixel and its related searching direction in FCMI; and (c) final extended change region.

\section{Experiment}

In this section, the proposed system was investigated by four experiments based on four image scenes depicting the different land cover change events. Three widely used methods, namely, change detection based on PCA and K-means (CD_PCA_Kmeans) [1], multiresolution level set change detection (MLS_CD) [2], and a semi-supervised fuzzy cluster means change detection method (Semi_FCM) [3], were compared with the proposed framework to evaluate the effectiveness of the proposed system. 


\subsection{Dataset Description}

The first dataset: The two images used in our experiments are acquired by using Zeiss RMK TOP 15 Aerial Survey Camera System at a flying height of approximately $2400 \mathrm{~m}$ in April 2007 and July 2014. The two RGB 24-bit images describe a landslide event in Hong Kong, as depicted in Figure 8. The image is $754 \times 694$ pixels with a $0.5 \mathrm{~m}$ spatial resolution. The ground truth reference map was interpreted manually and is displayed in Figure $8 \mathrm{~d}$ to compare the proposed system with other methods quantitatively.

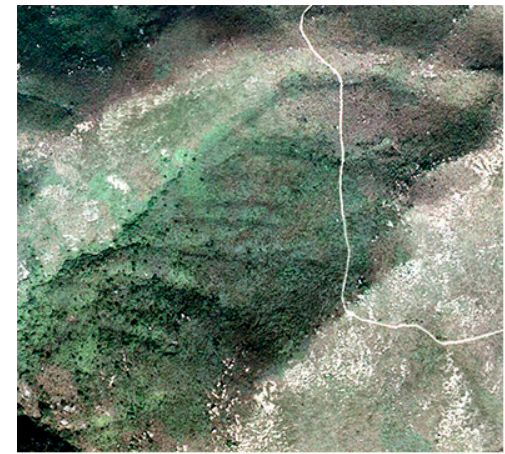

(a)

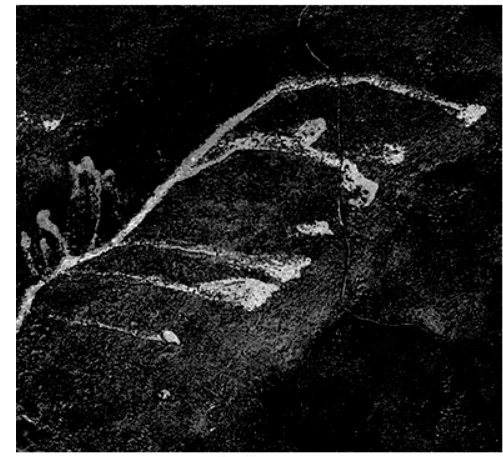

(c)

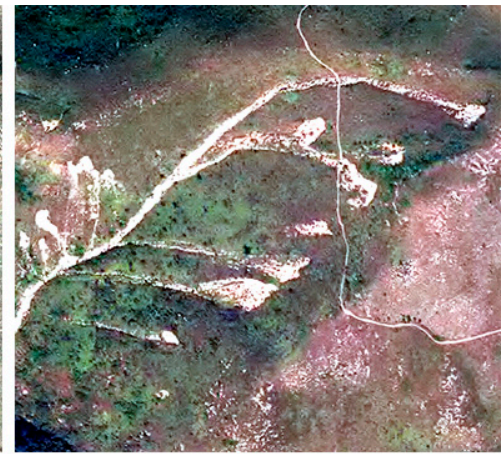

(b)

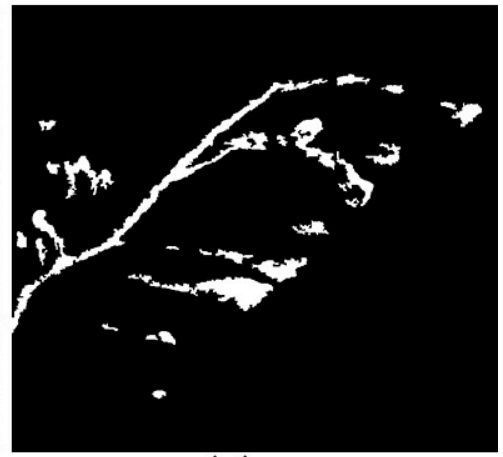

(d)

Figure 8. First dataset: Digital Orthophoto Map acquired by an aerial plane in: (a) April 2007; and (b) July 2014; (c) corresponding change magnitude image (CMI) generated through the difference method; and (d) reference of the changed area caused by the landslide.

The second dataset: This dataset was captured similar to the first dataset, which also has a spatial resolution of $0.5 \mathrm{~m}$ per pixel. The size of the study area is $750 \times 950$ pixels. The pre- and post-event images are presented in Figure 9a,b. The ground truth is also interpreted manually and illustrated in Figure $9 \mathrm{~d}$. This area is covered by many kinds of grass and a spot of shrubs and trees. The spectral heterogeneity of these bi-temporal images is relatively high.

The third dataset: This dataset is open data for change detection evaluation. It is composed of two 8-bit images acquired by Landsat thematic mapper sensor of the Landsat-7 satellite in Mexico area in April 2000 and May 2002. From the entire scene, a section of $512 \times 512$ pixels was selected as the test site. Figure 10a,b depicts the channel 4 images in 2000 and 2002, respectively. Comparing the two images, fire destroyed a large portion of vegetation in the considered changed region. The reference change map was obtained manually to obtain a quantitative evaluation, as presented in Figure 10d. 


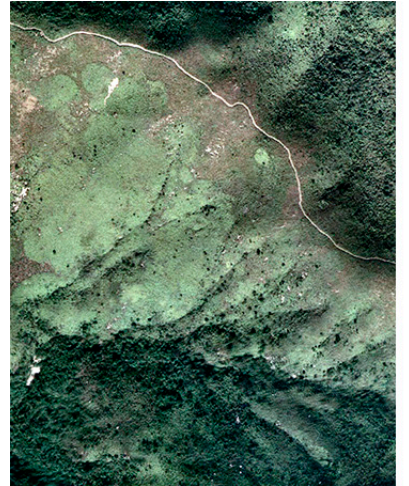

(a)

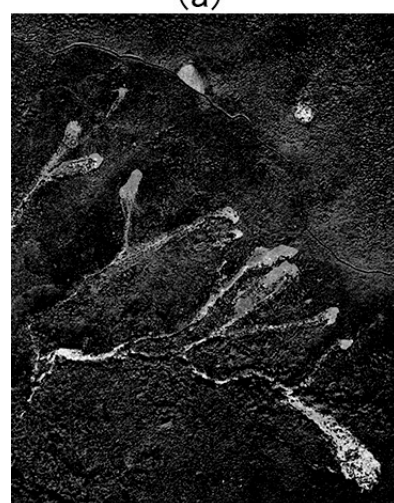

(c)

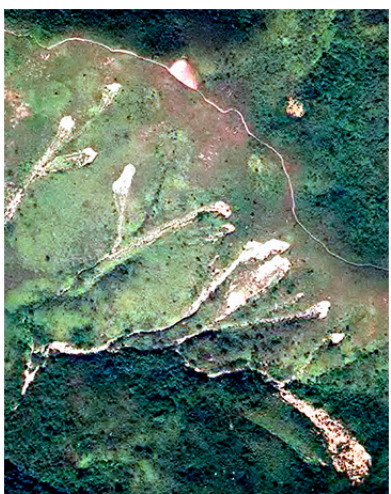

(b)

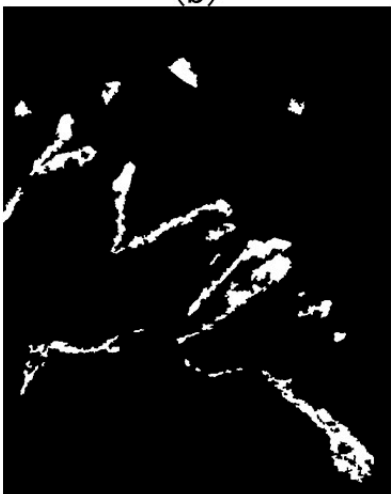

(d)

Figure 9. Second dataset: Digital Orthophoto Map acquired by an aerial plane in: (a) April 2007; and (b) July 2014; (c) corresponding CMI generated through the difference method; and (d) reference of the changed area caused by the landslide.

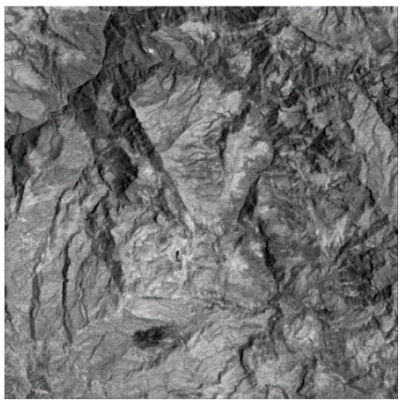

(a)

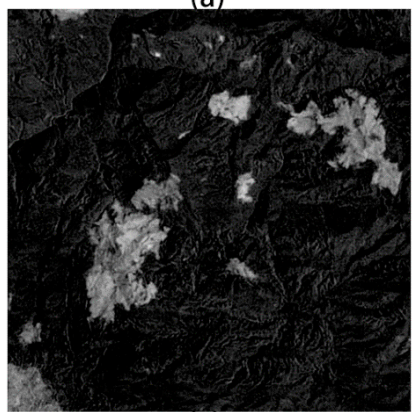

(c)

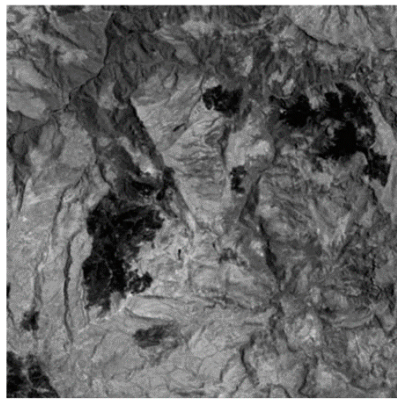

(b)

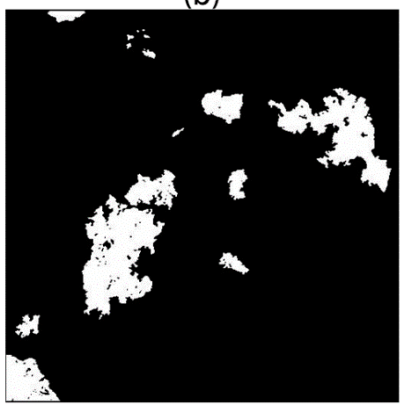

(d)

Figure 10. Third dataset: Mexico dataset, band 4 of the Landsat TM image captured in (a) April 2000; and (b) May 2002; (c) corresponding CMI generated through the difference method; and (d) reference of the changed area. 
The fourth dataset: This dataset is composed of two 8-bit images acquired by the Landsat TM sensor of the Landsat-5 satellite on September 1995 and July 1996. The size of the testing site is $412 \times 300$. pixels, which covers Lake Mulargia on Sardinia Island (Italy). The water level in the lake varied obviously between the two aforementioned acquisition dates. Figure 11a,b depicts the band 4 images in 1995 and 1996, correspondingly. In addition, the reference map is defined manually according to the detailed visual analysis based on bi-temporal image comparison.

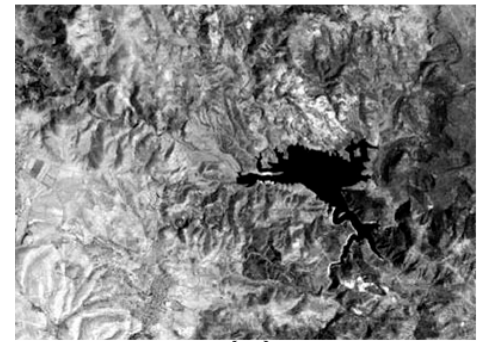

(a)

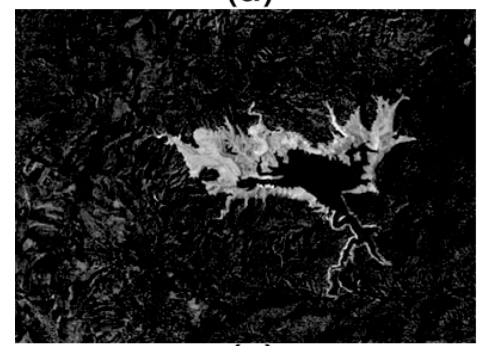

(c)

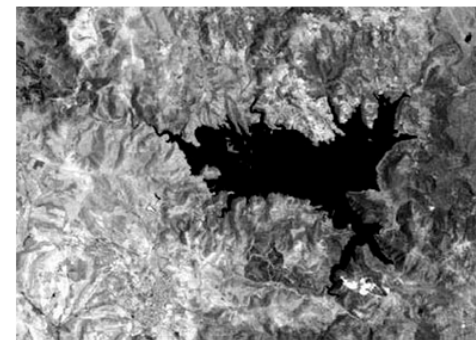

(b)

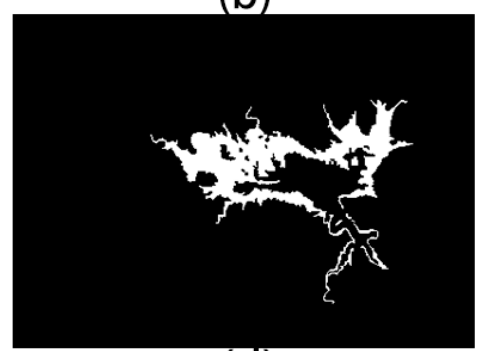

(d)

Figure 11. Fourth dataset: Sardinia Island (Italy) dataset, band 4 of the Landsat TM image captured in (a) September 1995; and (b) July 1996; (c) corresponding CMI generated through the difference method; and (d) reference of the changed area.

\subsection{Experimental Setup and Parameter Setting}

The first and second experiments were designed to test the effectiveness of the proposed framework for LCCD using aerial images with high spatial resolution. Therefore, the aerial images with a $0.5 \mathrm{~m}$ resolution were adopted in the two experiments, as depicted in Figure 8a,b and Figure 9a,b. In the two experiments, the proposed framework was compared with CD_PCA_Kmeans [1], CD_MLS [2], and Semi_FCM [3]. The optimal parameters of each experiment were obtained by the trial-and-error method. The parameter details of each approach are provided as follows: (1) in the CD_PCA_Kmeans approach, two parameters $(\mathrm{h} \times \mathrm{h}$ nonoverlapping blocks and s orthonormal eigenvectors) were set to $\mathrm{h}=6$ and $\mathrm{s}=5$; (2) for the previous CD_MLS approach, the regularization parameter of the model is $\mu=0.1$ and $\mathrm{L}=2$; and (3) the parameter of the Semi_FCM change detection approach was set to $\alpha=8$. The binary threshold of the proposed framework is $\mathrm{T}=43$ and $\mathrm{T}=46$ for the first and second experiments, respectively.

In addition, the adaptability of the proposed framework for remote sensing images with the median-low resolution was tested in the third and fourth experiments. Two open datasets for change detection, such as the Mexico and Sardinia Island (Italy) datasets, were used in the two experiments, as displayed in Figures 10 and 11. For the two previous experiments, the proposed framework was compared with CD_PCA_Kmeans [1], CD_MLS [2], and Semi_FCM [3]. The parameters of these approaches were provided in detail as follows: (1) in the CD_PCA_Kmeans approach, two parameters were set at $\mathrm{h}=4$ and $\mathrm{s}=3$; (2) for the CD_MLS approach, the regularization parameter of the model was $\mu=0.1$ and $\mathrm{L}=2$; and (3) the parameter of Semi_FCM change detection approach was set at $\alpha=8$. The parameter of the proposed framework was $\mathrm{T}=30$ and $\mathrm{T}=52$ for the third and fourth experiments, respectively. The ground truth for the two datasets is displayed in Figures 10d and 11d. 


\subsection{Results and Quantitative Evaluation}

Three quantitative evaluation indices, false alarm (FA), missed alarm (MA), and overall error (OE), are used for experimental comparisons to evaluate the proposed framework quantitatively [64]. In this process, UC is the number of changed pixels that are actually unchanged pixels in the CDM when compared with the ground truth. TRU is the number of pixels that are unchanged when compared with the ground truth; $\mathrm{CU}$ is the unchanged pixels in the CDM but is changed pixels when compared to the ground truth. TRC is the total number of changed pixels in the ground truth. Therefore, the equations of FA, MA, and OE can be expressed as FA $=\frac{U C}{T R U} \times 100 \%, \mathrm{MA}=\frac{C U}{T R C} \times 100 \%$, and $\mathrm{OE}=\frac{U C+C U}{T R C+T R U} \times 100 \%$, respectively.

The first image scene depicted a landslide event in Hong Kong, as illustrated in Figure 8a,b. Comparison results are depicted in Figure 12, and quantitative comparisons are listed in details in Table 1. From the comparisons, the proposed framework performed better than CD_PCA_Kmeans [1], CD_MLS [2], and Semi_FCM [3]. In Table 1, the proposed framework was superior with regards to FA, $\mathrm{MA}$, and OE compared with the abovementioned methods. This condition revealed that the proposed framework is feasible and effective for LCCD using remote sensing images with high spatial resolution.
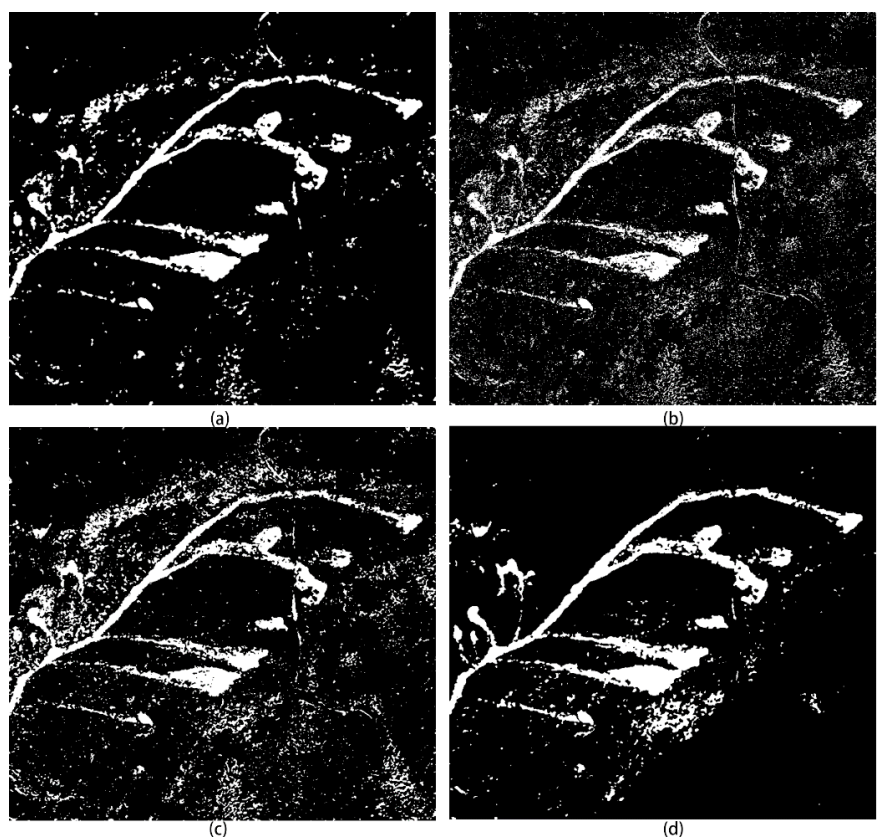

Figure 12. First dataset: CDMs obtained by: (a) CD_PCA_Kmeans [1]; (b) CD_MLS [2]; (c) Semi_FCM method [3]; and (d) the proposed framework.

Table 1. Change Detection Quantitative Evaluation (\%) on the First Dataset (more details can be tracked in Table S1.).

\begin{tabular}{cccc}
\hline Method & False Alarm & Missed Alarm & Overall Errors \\
\hline CD_PCA_Kmeans & 4.34 & 12.46 & 4.76 \\
CD_MLS & 7.6 & 14.55 & 7.95 \\
Semi_FCM & 8.71 & 9.19 & 8.73 \\
The proposed & 3.29 & 7.03 & 3.48 \\
\hline
\end{tabular}

Two aerial images for the second experiment were used for investigating the effectiveness of the proposed framework, as depicted in Figure 9a,b. From the comparison in Figure 13 and Table 2, the framework achieved the best accuracy in terms of FA and OE compared with the results of CD_PCA_Kmeans [1] and CD_MLS [2], although the Semi_FCM [3] obtained the best accuracy in 
terms of MA. Overall, the proposed framework was superior to the compared methods in terms of FA and OE. The proposed framework can achieve CDM accurately with low FA, although it relatively missed many changed pixels.

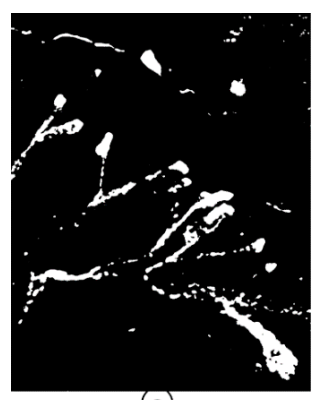

(a)

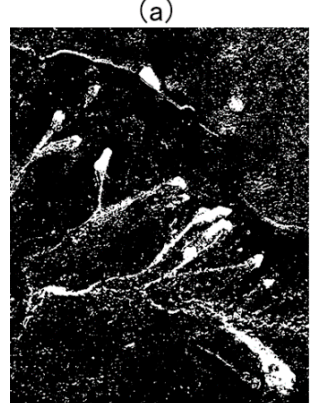

(c)

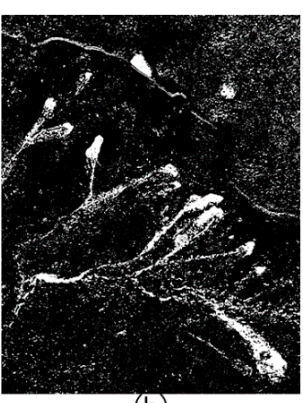

(b)

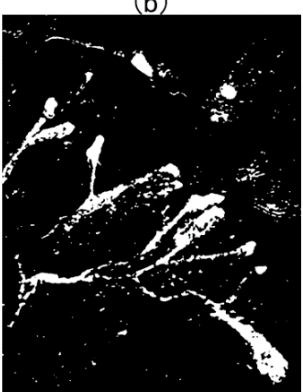

(d)

Figure 13. Second dataset: CDMs obtained by: (a) CD_PCA_Kmeans [1]; (b) CD_MLS [2]; (c) Semi_FCM method [3]; and (d) the proposed framework.

Table 2. Change Detection Quantitative Evaluation (\%) on the Second Dataset (more details can be tracked in Table S2.).

\begin{tabular}{cccc}
\hline Method & False Alarm & Missed Alarm & Overall Errors \\
\hline CD_PCA_kmeans & 2.52 & 19.47 & 3.35 \\
CD_MLS & 7.48 & 20.8 & 8.13 \\
Semi_FCM & 8.53 & 15.16 & 8.86 \\
The proposed & 1.87 & 17.90 & 2.66 \\
\hline
\end{tabular}

In the third experiment, the effectiveness of the proposed framework was evaluated by comparing the different change detection methods for the Mexico datasets, including CD_PCA_Kmeans [1], CD_MLS [2], and Semi_FCM [3]. The spatial resolution of this dataset was $30 \mathrm{~m}$ per pixel. The comparison results are depicted in Figure 14 and Table 3. From the comparison, the proposed framework obtained superior MA and OE but recorded insufficient FA. However, the proposed framework can achieve the balance between the three measurements (MA, OE, and FA) and a relatively satisfactory CDM.

Table 3. Change Detection Quantitative Evaluation (\%) on the Mexico Dataset (more details can be tracked in Table S3.).

\begin{tabular}{cccc}
\hline Method & False Alarm & Missed Alarm & Overall Errors \\
\hline CD_PCA_Kmeans & 0.78 & 10.3 & 1.71 \\
CD_MLS & 0.58 & 11.9 & 1.68 \\
Semi_FCM & 0.41 & 15.0 & 1.83 \\
The proposed & 1.08 & 4.73 & 1.43 \\
method & & & \\
\hline
\end{tabular}




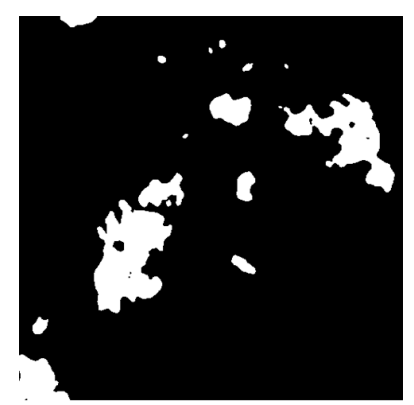

(a)

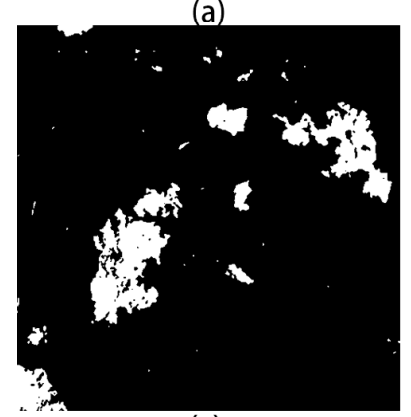

(c)

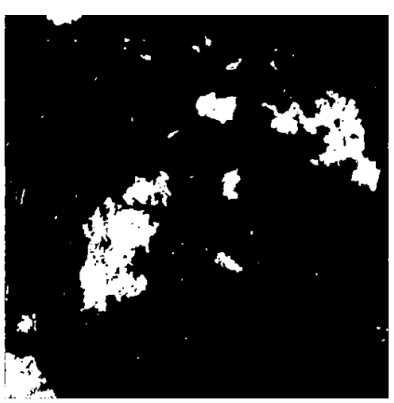

(b)

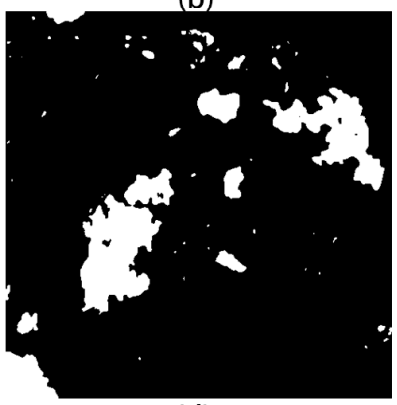

(d)

Figure 14. Mexico dataset: CDMs obtained by: (a) CD_PCA_Kmeans [1]; (b) CD_MLS [2]; (c) Semi_FCM method [3]; and (d) the proposed framework.

The fourth experiment was designed to test the effectiveness and adaptability of the proposed framework when using the remote sensing images of median-low spatial resolution. The Sardinia Island datasets with a pixel resolution of $30 \mathrm{~m}$ were adopted in this experiment. The comparison results are presented in Figure 15 and Table 4. The CDM obtained by the proposed framework recorded less noise and performed better than the CDM of CD_PCA_Kmeans [1], CD_MLS [2], and Semi_FCM [3]. In Table 4, the proposed framework for change detection missed the least pixels, and its total error is the lowest. The FA of the proposed framework is $1.48 \%$, which is adjacent to the $1.26 \%$ obtained by CD_PCA_Kmeans [1], although the best FA performance was achieved by CD_PCA_Kmeans [1]. Furthermore, the proposed system achieved the best change detection accuracy in terms of MA and OE for the Sardinia Island dataset.

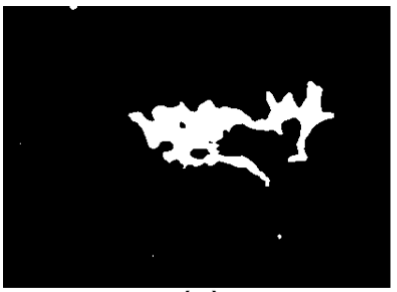

(a)

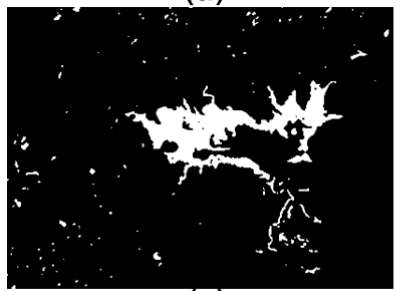

(c)

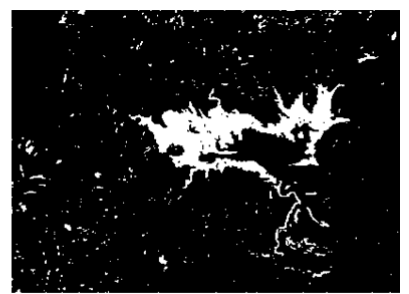

(b)

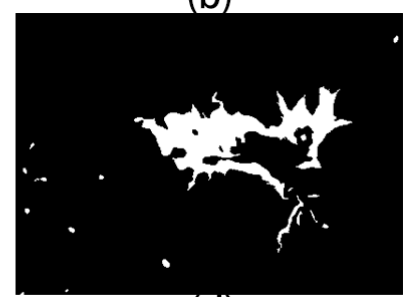

(d)

Figure 15. Sardinia Island dataset: CDMs obtained by: (a) CD_PCA_Kmeans [1]; (b) CD_MLS [2]; (c) Semi_FCM method [3]; and (d) the proposed framework. 
Table 4. Change Detection Quantitative Evaluation (\%) on the Sardinia Island dataset (more details can be tracked in Table S4.).

\begin{tabular}{cccc}
\hline Method & False Alarm & Missed Alarm & Overall Errors \\
\hline CD_PCA_Kmeans & 1.26 & 14.9 & 2.1 \\
CD_MLS & 2.64 & 9.83 & 3.09 \\
Semi_FCM & 1.77 & 9.86 & 2.27 \\
The proposed & 1.48 & 7.93 & 1.88 \\
\hline
\end{tabular}

\section{Discussion}

The proposed framework notably improved the detection accuracy in the performance and quantitative comparisons of the experiments. Furthermore, the experimental results demonstrated that: (1) the proposed framework can achieve an enhanced change detection performance with high accuracies; and (2) the proposed framework is generally suitable for aerial images with high spatial resolution and satellite images with median-low spatial resolution. The advantages of the proposed framework indicated considerable potential applications in LCCD using remote sensing images compared with the widely used methods, such as the related methods in the experiments.

In addition, the parameter setting is more convenient in the proposed framework than in CD_PCA_PCA [1], CD_MLS [2], and Semi_FCM [3]. One free-parameter is required in the proposed framework. Furthermore, the second technique of the proposed framework automatically provided a range for its parameter determination, which is valuable for parameter selection, especially for an inexperienced practitioner. In Figure 16, the pairwise green line marked the threshold range predicted by the proposed framework for each experiment. The first minimum and maximum were captured automatically and referenced to the prediction threshold range. The binary threshold could be determined easily with the suggested auxiliary range, although the predicted range cannot directly determine a rational binary threshold for dividing a CMI into a BCDM. Furthermore, the relationship between detection accuracy and parameters varied within the suggested range, as illustrated in Figure 17. MA is increased with the increment of parameter $(\mathrm{T})$ in each experiment. By contrast, FA and $\mathrm{OE}$ are decreased with the increment in parameter $(\mathrm{T})$ in each experiment. Furthermore, FA and $\mathrm{OE}$ inclined gradually to the horizontal line with the increment in parameter $(\mathrm{T})$ and the constraints of the predicted range. This finding is essential for the parameter determination of the proposed framework.

The growth performance of the third proposed technique, namely, RGA, is illustrated in Figure 18 to demonstrate the effectiveness of this method. In Figure 18, the pixels of the growing area were along the change area and were continuously spatial despite the small size of the growing area. The quantitative comparison between the results of the second and third techniques (RGA) is presented in Table 5; this comparison was performed to investigate the advantages of the proposed method. In Table 5, the detection accuracy was relatively improved in terms of the four experimental datasets when the proposed RGA is applied to optimize the initial binary change detection result, which was obtained using the second technique.

Table 5. Quantitative Comparison (\%) between the Second and Third Techniques on the Four Datasets.

\begin{tabular}{ccccccc}
\hline \multirow{2}{*}{ Dataset } & \multicolumn{2}{c}{ Binary Map Obtained by the Second Technique } & \multicolumn{3}{c}{ Binary Map Optimized by the Third Technique } \\
\cline { 2 - 7 } & False Alarm & Missed Alarm & Overall Errors & False Alarm & Missed Alarm & Overall Errors \\
\hline First Data & 3.85 & 8.40 & 4.30 & 3.29 & 7.03 & 3.48 \\
Second Data & 2.21 & 20.92 & 3.10 & 1.87 & 17.9 & 2.66 \\
Mexico & 1.12 & 5.23 & 2.24 & 1.08 & 7.73 & 7.93 \\
Sardinia Island & 1.51 & 10.20 & 2.00 & 1.48 & 1.83 \\
\hline
\end{tabular}



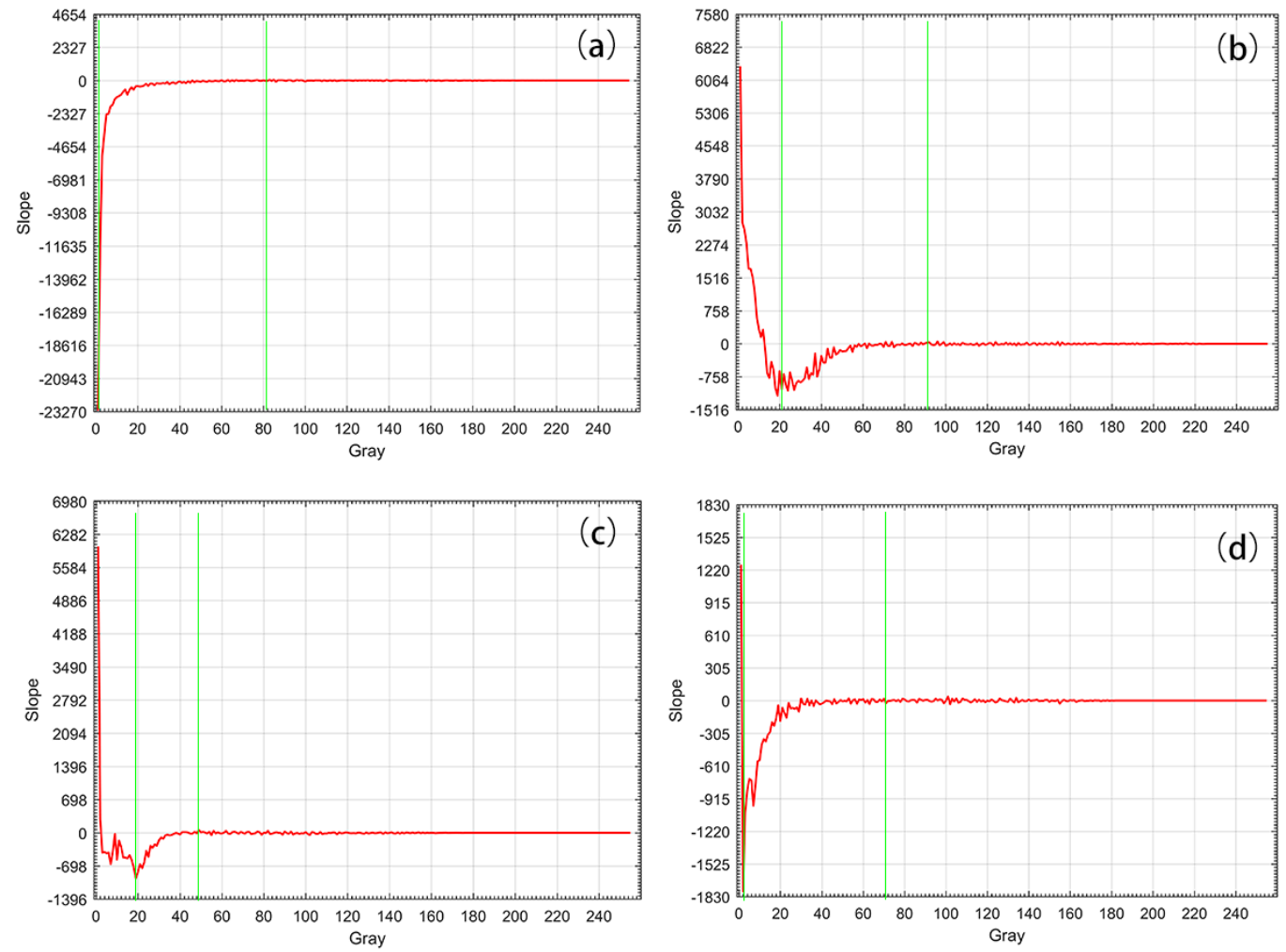

Figure 16. TRP using the proposed framework for each experiment; the predicted ranges are: (a) $[0,84]$; (b) $[26,91]$; (c) $[18,48]$; and (d) $[0,72]$ for each experiment.

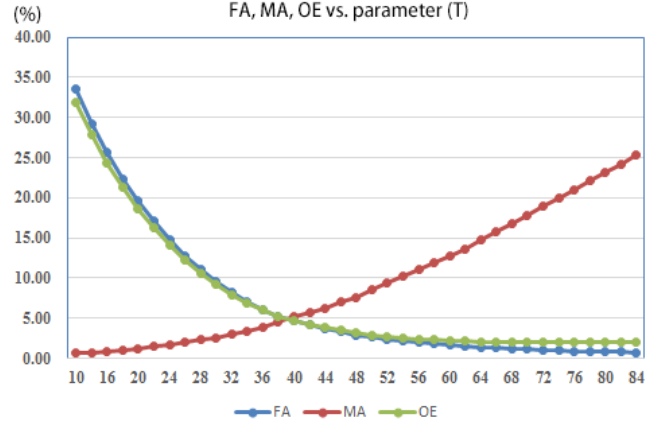

(a)

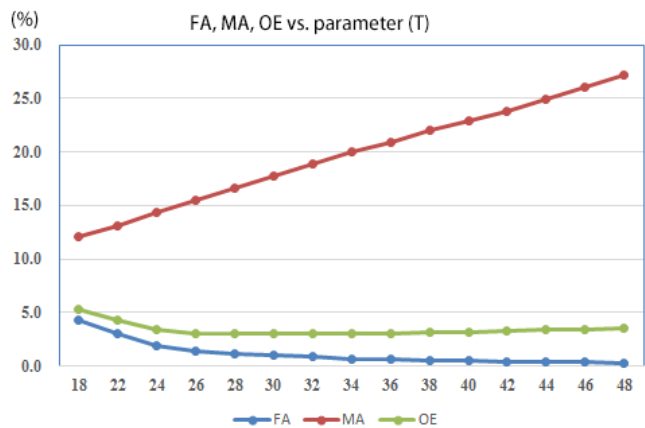

(c)

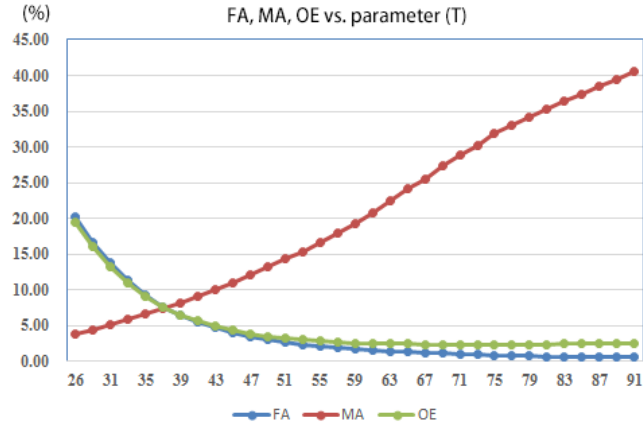

(b)

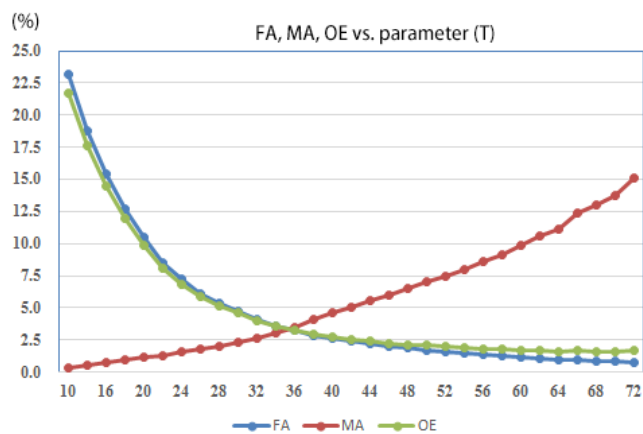

(d)

Figure 17. Relationship between detection accuracy and parameter $(\mathrm{T})$ setting of the proposed framework in each experiment: (a) Experiment 1; (b) Experiment 2; (c) Experiment 3; and (d) Experiment 4. 

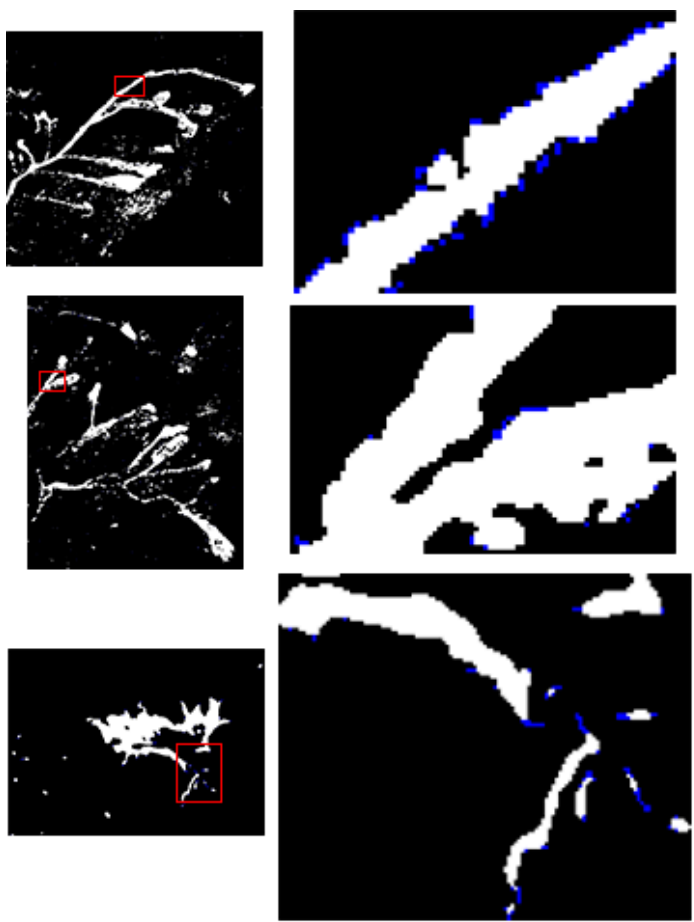

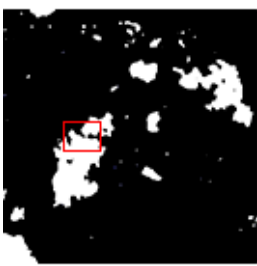

(a)

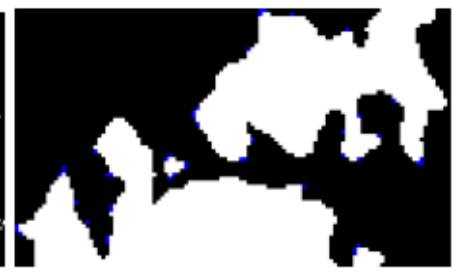

(b)

Figure 18. Growth performance based on the third technique (RGA) used to observe the details of the growth performance: (a) the selected observed range; and (b) the corresponding zoomed in figure. Blue, white, and black pixels represent the growing area, change region, and background, respectively.

\section{Conclusions}

In this work, a semi-automatic change detection framework is proposed for LCCD. The proposed framework consists of three techniques for improving the performance of change detection and promoting the automation degree for practical applications. The first technique is the automatic noise reduction based on GF, which aims to reduce the noise in a prepared CMI. Then, the TRP strategy is proposed as the second technique to improve the automation degree and assist the parameter determination of the proposed framework. Finally, the modified RGA is suggested as the third technique for improving the detection accuracy because missing alarm pixels typically distribute around a change region when a binary threshold is divided from the FCMI into the BCDM.

Four experiments were conducted on four remote sensing image scenes that are related to different actual land cover change events, including landslides and fire disasters. The experimental results demonstrate the effectiveness of the proposed framework and obtained superior detection results compared with widely used methods, such as CD_PCA_Kmeans [1], CD_MLS [2], and Semi_FCM [3]. The advantages of the proposed framework can be summarized as follows: (1) the proposed framework is favorable in terms of change detection accuracy and performance; (2) the proposed framework is suitable for detecting land cover change using the remote sensing images with high and median-low spatial resolutions based on the experiment results because the detection procedure of the proposed framework is data-independent; and (3) parameter setting requires minimal tuning because it only maintains one free parameter. Furthermore, the free parameter can be determined through 
a procedure assisted by a predicted range. Overall, the superiority of the proposed framework indicates considerable potential applications for LCCD using remote sensing images.

In the future study, an extensive investigation of the proposed system will be conducted on additional types of remote sensing images, such as unmanned aerial vehicle image with very high spatial resolution. Theoretically, further investigations conducted through a method with different sourcing images will enhance the robustness of the method. Furthermore, additional investigation will broaden the use and applicability of the proposed method.

Supplementary Materials: The following are available online at www.mdpi.com/2072-4292/9/11/1112/s1, Table S1: Confusion Matrix and Change Detection Quantitative Evaluation (\%) on the First Dataset; Table S2: Confusion Matrix and Change Detection Quantitative Evaluation (\%) on the Second Dataset; Table S3: Confusion Matrix and Change Detection Quantitative Evaluation (\%) on the Mexico Dataset; Table S4: Confusion Matrix and Change Detection Quantitative Evaluation (\%) on the Sardinia Island Dataset.

Acknowledgments: The authors thank the Editor-in-Chief, associate editor, and reviewers for their insightful comments and suggestions. The authors thank LiChen Yang for his program in the experiments. This work was supported by the project Dynamic Characterization of Smart City (1-ZE24), the National Science Foundation China (61701396), the Key Laboratory for National Geographic Census and Monitoring, National Administration of Surveying, Mapping, and Geoinformation (2015NGCM), key Laboratory of Spatial Data Mining \& Information Sharing of Ministry of Education, Fuzhou University (2018LSDMIS01), and the project from the China Postdoctoral Science Foundation (2015M572658XB).

Author Contributions: ZhiYong Lv was primarily responsible for the original idea and experimental design. WenZhong Shi provided important suggestions for improving the paper's quality. Jón Atli Benediktsson provided ideas to improve the quality of the paper. XiaoCheng Zhou provided contributions to improve the quality of the paper in the revision procedure.

Conflicts of Interest: The authors declare no conflict of interest.

\section{References}

1. Celik, T. Unsupervised change detection in satellite images using principal component analysis and k-means clustering. IEEE Geosci. Remote Sens. Lett. 2009, 6, 772-776. [CrossRef]

2. Bazi, Y.; Melgani, F.; Al-Sharari, H.D. Unsupervised change detection in multispectral remotely sensed imagery with level set methods. IEEE Trans. Geosci. Remote Sens. 2010, 48, 3178-3187. [CrossRef]

3. Shao, P.; Shi, W.; He, P.; Hao, M.; Zhang, X. Novel approach to unsupervised change detection based on a robust semi-supervised fcm clustering algorithm. Remote Sens. 2016, 8, 264. [CrossRef]

4. Giustarini, L.; Hostache, R.; Matgen, P.; Schumann, G.J.-P.; Bates, P.D.; Mason, D.C. A change detection approach to flood mapping in urban areas using terrasar-x. IEEE Trans. Geosci. Remote Sens. 2013, 51, 2417-2430. [CrossRef]

5. Jin, S.; Yang, L.; Danielson, P.; Homer, C.; Fry, J.; Xian, G. A comprehensive change detection method for updating the national land cover database to circa 2011. Remote Sens. Environ. 2013, 132, 159-175. [CrossRef]

6. Beuchle, R.; Grecchi, R.C.; Shimabukuro, Y.E.; Seliger, R.; Eva, H.D.; Sano, E.; Achard, F. Land cover changes in the brazilian cerrado and caatinga biomes from 1990 to 2010 based on a systematic remote sensing sampling approach. Appl. Geogr. 2015, 58, 116-127. [CrossRef]

7. Homer, C.G.; Dewitz, J.A.; Yang, L.; Jin, S.; Danielson, P.; Xian, G.; Coulston, J.; Herold, N.D.; Wickham, J.; Megown, K. Completion of the 2011 national land cover database for the conterminous united states-representing a decade of land cover change information. Photogramm. Eng. Remote Sens. 2015, 81, 345-354.

8. Li, Z.; Shi, W.; Lu, P.; Yan, L.; Wang, Q.; Miao, Z. Landslide mapping from aerial photographs using change detection-based markov random field. Remote Sens. Environ. 2016, 187, 76-90. [CrossRef]

9. Li, Z.; Shi, W.; Myint, S.W.; Lu, P.; Wang, Q. Semi-automated landslide inventory mapping from bitemporal aerial photographs using change detection and level set method. Remote Sens. Environ. 2016, 175, 215-230. [CrossRef]

10. Yang, H.; Dou, A.; Zhang, W.; Huang, S. Study on Extraction of Earthquake Damage Information Based on Regional Optimizing Change Detection from Remote Sensing Image. In Proceedings of the IEEE International Geoscience and Remote Sensing Symposium (IGARSS), Quebec, QC, Canada, 13-18 July 2014; pp. 4272-4275. 
11. Radke, R.J.; Andra, S.; Al-Kofahi, O.; Roysam, B. Image change detection algorithms: A systematic survey. IEEE Trans. Image Process. 2005, 14, 294-307. [CrossRef] [PubMed]

12. Hussain, M.; Chen, D.; Cheng, A.; Wei, H.; Stanley, D. Change detection from remotely sensed images: From pixel-based to object-based approaches. ISPRS 2013, 80, 91-106. [CrossRef]

13. Falco, N.; Dalla Mura, M.; Bovolo, F.; Benediktsson, J.A.; Bruzzone, L. Change detection in vhr images based on morphological attribute profiles. IEEE Geosci. Remote Sens. Lett. 2013, 10, 636-640. [CrossRef]

14. Bruzzone, L.; Prieto, D.F. Automatic analysis of the difference image for unsupervised change detection. IEEE Trans. Geosci. Remote Sens. 2000, 38, 1171-1182. [CrossRef]

15. Chen, J.; Lu, M.; Chen, X.; Chen, J.; Chen, L. A spectral gradient difference based approach for land cover change detection. ISPRS 2013, 85, 1-12. [CrossRef]

16. Chen, J.; Chen, X.; Cui, X.; Chen, J. Change vector analysis in posterior probability space: A new method for land cover change detection. IEEE Geosci. Remote Sens. Lett. 2011, 8, 317-321. [CrossRef]

17. Ghosh, A.; Mishra, N.S.; Ghosh, S. Fuzzy clustering algorithms for unsupervised change detection in remote sensing images. Inf. Sci. 2011, 181, 699-715. [CrossRef]

18. Yuan, F.; Sawaya, K.E.; Loeffelholz, B.C.; Bauer, M.E. Land cover classification and change analysis of the twin cities (minnesota) metropolitan area by multitemporal landsat remote sensing. Remote Sens. Environ. 2005, 98, 317-328. [CrossRef]

19. Lhermitte, S.; Verbesselt, J.; Verstraeten, W.W.; Coppin, P. A comparison of time series similarity measures for classification and change detection of ecosystem dynamics. Remote Sens. Environ. 2011, 115, 3129-3152. [CrossRef]

20. Chen, X.; Chen, J.; Shi, Y.; Yamaguchi, Y. An automated approach for updating land cover maps based on integrated change detection and classification methods. ISPRS 2012, 71, 86-95. [CrossRef]

21. Zhu, Z.; Woodcock, C.E. Continuous change detection and classification of land cover using all available landsat data. Remote Sens. Environ. 2014, 144, 152-171. [CrossRef]

22. Seebach, L.; Strobl, P.; Vogt, P.; Mehl, W.; San-Miguel-Ayanz, J. Enhancing post-classification change detection through morphological post-processing-a sensitivity analysis. Int. J. Remote Sens. 2013, 34, 7145-7162. [CrossRef]

23. Lu, D.; Mausel, P.; Brondizio, E.; Moran, E. Change detection techniques. Int. J. Remote Sens. 2004, 25, 2365-2401. [CrossRef]

24. Huang, X.; Zhang, L.; Zhu, T. Building change detection from multitemporal high-resolution remotely sensed images based on a morphological building index. IEEE J. Sel. Top. Appl. Earth Obs. Remote Sens. 2014, 7, 105-115. [CrossRef]

25. Huang, X.; Zhang, L. An svm ensemble approach combining spectral, structural, and semantic features for the classification of high-resolution remotely sensed imagery. IEEE Trans. Geosci. Remote Sens. 2013, 51, 257-272. [CrossRef]

26. Subudhi, B.N.; Bovolo, F.; Ghosh, A.; Bruzzone, L. Spatio-contextual fuzzy clustering with markov random field model for change detection in remotely sensed images. Opt. Laser Technol. 2014, 57, 284-292. [CrossRef]

27. Gu, W.; Lv, Z.; Hao, M. Change detection method for remote sensing images based on an improved markov random field. Multimed. Tools Appl. 2017, 76, 17719-17734. [CrossRef]

28. Ghosh, A.; Subudhi, B.N.; Bruzzone, L. Integration of gibbs markov random field and hopfield-type neural networks for unsupervised change detection in remotely sensed multitemporal images. IEEE Trans. Image Process. 2013, 22, 3087-3096. [CrossRef] [PubMed]

29. Volpi, M.; Tuia, D.; Bovolo, F.; Kanevski, M.; Bruzzone, L. Supervised change detection in vhr images using contextual information and support vector machines. Int. J. Appl. Earth Obs. Geoinf. 2013, 20, 77-85. [CrossRef]

30. Lv, Z.Y.; Zhang, P.; Benediktsson, J.A.; Shi, W.Z. Morphological profiles based on differently shaped structuring elements for classification of images with very high spatial resolution. IEEE J. Sel. Top. Appl. Earth Obs. Remote Sens. 2014, 7, 4644-4652. [CrossRef]

31. Huang, X.; Zhang, L.; Li, P. An adaptive multiscale information fusion approach for feature extraction and classification of ikonos multispectral imagery over urban areas. IEEE Geosci. Remote Sens. Lett. 2007, 4, 654-658. [CrossRef] 
32. Lv, Z.; Zhang, P.; Atli Benediktsson, J. Automatic object-oriented, spectral-spatial feature extraction driven by tobler's first law of geography for very high resolution aerial imagery classification. Remote Sens. 2017, 9, 285. [CrossRef]

33. Topouzelis, K.; Papakonstantinou, A.; Pavlogeorgatos, G. Coastline change detection using uav, Remote Sensing, GIS and 3D reconstruction. In Proceedings of the 5th International Conference on Environmental Management, Engineering, Planning and Economics (CEMEPE) and SECOTOX Conference, Mykonos Island, Greece, 14-18 June 2015.

34. Tewkesbury, A.P.; Comber, A.J.; Tate, N.J.; Lamb, A.; Fisher, P.F. A critical synthesis of remotely sensed optical image change detection techniques. Remote Sens. Environ. 2015, 160, 1-14. [CrossRef]

35. Liu, S.; Bruzzone, L.; Bovolo, F.; Du, P. Hierarchical unsupervised change detection in multitemporal hyperspectral images. IEEE Trans. Geosci. Remote Sens. 2015, 53, 244-260.

36. Wen, D.; Huang, X.; Zhang, L.; Benediktsson, J.A. A novel automatic change detection method for urban high-resolution remotely sensed imagery based on multiindex scene representation. IEEE Trans. Geosci. Remote Sens. 2016, 54, 609-625. [CrossRef]

37. Dalla Mura, M.; Benediktsson, J.A.; Bovolo, F.; Bruzzone, L. An unsupervised technique based on morphological filters for change detection in very high resolution images. IEEE Geosci. Remote Sens. Lett. 2008, 5, 433-437. [CrossRef]

38. Wu, H.; Li, Z.-L. Scale issues in remote sensing: A review on analysis, processing and modeling. Sensors 2009, 9, 1768-1793. [CrossRef] [PubMed]

39. Woodcock, C.E.; Strahler, A.H. The factor of scale in remote sensing. Remote Sens. Environ. 1987, 21, $311-332$. [CrossRef]

40. Townshend, J.R.; Justice, C.O.; Gurney, C.; McManus, J. The impact of misregistration on change detection. IEEE Trans. Geosci. Remote Sens. 1992, 30, 1054-1060. [CrossRef]

41. Marchesi, S.; Bovolo, F.; Bruzzone, L. A context-sensitive technique robust to registration noise for change detection in vhr multispectral images. IEEE Trans. Image Process. 2010, 19, 1877-1889. [CrossRef] [PubMed]

42. Cheng, K.; Wei, C.; Chang, S. Locating landslides using multi-temporal satellite images. Adv. Space Res. 2004, 33, 296-301. [CrossRef]

43. Gong, M.; Zhan, T.; Zhang, P.; Miao, Q. Superpixel-based difference representation learning for change detection in multispectral remote sensing images. IEEE Trans. Geosci. Remote Sens. 2017, 55, 2658-2673. [CrossRef]

44. Lee, J.-H.; Biging, G.S.; Fisher, J.B. An individual tree-based automated registration of aerial images to lidar data in a forested area. Photogramm. Eng. Remote Sens. 2016, 82, 699-710. [CrossRef]

45. Zhang, L.; Li, A.; Zhang, Z.; Yang, K. Global and local saliency analysis for the extraction of residential areas in high-spatial-resolution remote sensing image. IEEE Trans. Geosci. Remote Sens. 2016, 54, 3750-3763. [CrossRef]

46. Lv, Z.; Zhang, X.; Benediktsson, J.A. Developing a general post-classification framework for land-cover mapping improvement using high-spatial-resolution remote sensing imagery. Remote Sens. Lett. 2017, 8, 607-616. [CrossRef]

47. Heijden, K.V.D. Image Based Measurement Systems; Fraunhofer IOSB: Karlsruhe, Germany, 2007.

48. Sezgin, M. Survey over image thresholding techniques and quantitative performance evaluation. J. Electron. Imaging 2004, 13, 146-168.

49. Otsu, N. A threshold selection method from gray-level histograms. IEEE Trans. Syst. Man Cybern. 1979, 9, 62-66. [CrossRef]

50. Rosin, P.L.; Ioannidis, E. Evaluation of global image thresholding for change detection. Pattern Recognit. Lett. 2003, 24, 2345-2356. [CrossRef]

51. Alesheikh, A.A.; Ghorbanali, A.; Nouri, N. Coastline change detection using remote sensing. Int. J. Environ. Sci. Technol. 2007, 4, 61. [CrossRef]

52. Bovolo, F.; Bruzzone, L. A detail-preserving scale-driven approach to change detection in multitemporal sar images. IEEE Trans. Geosci. Remote Sens. 2005, 43, 2963-2972. [CrossRef]

53. Gong, M.; Su, L.; Jia, M.; Chen, W. Fuzzy clustering with a modified mrf energy function for change detection in synthetic aperture radar images. IEEE Trans. Fuzzy Syst. 2014, 22, 98-109. [CrossRef]

54. Levin, D. The approximation power of moving least-squares. Math. Comput. Am. Math. Soc. 1998, 67, 1517-1531. [CrossRef] 
55. Mao, F.; Gong, W.; Zhu, Z. Simple multiscale algorithm for layer detection with lidar. Appl. Opt. 2011, 50, 6591-6598. [CrossRef] [PubMed]

56. Shi, W.; Hao, M. Analysis of spatial distribution pattern of change-detection error caused by misregistration. Int. J. Remote Sens. 2013, 34, 6883-6897. [CrossRef]

57. Dai, X.; Khorram, S. The effects of image misregistration on the accuracy of remotely sensed change detection. IEEE Trans. Geosci. Remote Sens. 1998, 36, 1566-1577.

58. Kasetkasem, T.; Varshney, P.K. An image change detection algorithm based on markov random field models. IEEE Trans. Geosci. Remote Sens. 2002, 40, 1815-1823. [CrossRef]

59. Bruzzone, L.; Cossu, R. An adaptive approach to reducing registration noise effects in unsupervised change detection. IEEE Trans. Geosci. Remote Sens. 2003, 41, 2455-2465. [CrossRef]

60. Stauffer, M.; McKinney, R. Landsat Image Differencing as an Automated Land Cover Change Detection Technique; Computer Sciences Corp.: Silver Spring, MD, USA, 1978. Available online: https:/ /ntrs.nasa.gov/search. jsp?R=19830009647 (accessed on 10 September 2017).

61. Singh, A. Tropical Forest Monitoring Using Digital Landsat Data in Northeastern India; University of Reading: Reading, UK, 1984.

62. Fung, T.; LeDrew, E. The determination of optimal threshold levels for change detection using various accuracy indices. Photogramm. Eng. Remote Sens. 1988, 54, 1449-1454.

63. Krylov, V.A.; Moser, G.; Serpico, S.B.; Zerubia, J. False discovery rate approach to unsupervised image change detection. IEEE Trans. Image Process. 2016, 25, 4704-4718. [CrossRef] [PubMed]

64. Yetgin, Z. Unsupervised change detection of satellite images using local gradual descent. IEEE Trans. Geosci. Remote Sens. 2012, 50, 1919-1929. [CrossRef]

(C) 2017 by the authors. Licensee MDPI, Basel, Switzerland. This article is an open access article distributed under the terms and conditions of the Creative Commons Attribution (CC BY) license (http:/ / creativecommons.org/licenses/by/4.0/). 